\title{
Effects of the cognitive organization of route knowledge on judgments of macrospatial distance
}

\author{
GARY L. ALLEN and KATHLEEN C. KIRASIC \\ Old Dominion University, Norfolk, Virginia
}

\begin{abstract}
The effects of route segmentation were examined in a series of three experiments. Subjects in Experiment 1 divided an actual route into segments. Subjects in Experiment 2 performed corresponding proximity-judgment and distance-estimation tasks involving locations selected on the basis of the route segments identified in Experiment 1. Subjects in Experiment 3 performed a simple unidirectional distance-estimation task. Results from these experiments indicated that subjects can readily divide a route into segments and that these segments significantly bias judgments of macrospatial distance. These findings suggest a similarity between route segmentation in macrospatial cognition and categorization in other cognitive-task domains.
\end{abstract}

The study of macrospatial cognition, which is concerned with the acquisition and use of spatial knowledge of largescale environments, has attracted a great deal of interest from experimental psychologists recently. In some cases, this interest is motivated by the proposition that macrospatial cognitive processes-from recognition memory to spatial inference-making-are primitive or fundamental in terms of human cognitive development (see Fishbein, 1976; Piaget \& Inhelder, 1967; Siegel \& White, 1975; Stea, 1976). According to this proposition, empirical investigation of macrospatial cognition should lead to a more comprehensive understanding of human cognition in general. Although this view may be valid, it is also true that experimental research in macrospatial cognition stands to benefit greatly from empirical and conceptual advances made in the study of less fundamental, more abstract, nonspatial cognitive phenomena. Kuipers' (1978) description of route knowledge in terms of a production system (Newell \& Simon, 1972) and Stevens and Coupe's (1978) reference to hierarchically organized semantic networks (Norman, Rumelhart et al., 1975) in explaining systematic distortions of directional judgments serve as cases in point.

The concept of linear order representations, which emerged from studies of inference-making (e.g., Trabasso, 1977) and comparative judgments along unidimensional stimulus continua (e.g., Holyoak \& Mah, 1982; Kosslyn, Murphy, Bemesderfer, \& Feinstein, 1977; Moyer \& Dumais, 1978), is another theoretical construct with potential application to the study of macrospatial cognition. In the past, route knowledge has occasionally been referred to as a linear mode of spatial representation (Allen, 1981; Hazen, Lockman, \& Pick, 1978; Piaget, In-

Reprint requests should be directed to Gary L. Allen, Department of Psychology, Old Dominion University, Norfolk, VA 23508. helder, \& Szeminska, 1960), because it entails a sequentially ordered set of temporospatial relationships involving a mobile traveler within a macrospatial context (Allen, Siegel, \& Rosinski, 1978; Beck \& Wood, 1976; Moar \& Carleton, 1982). Of course, there are drastic differences between routes and the types of linear orders, such as the number line, the alphabet, and lists of words and nonsense syllables (Holyoak, 1978; Klahr, Chase, \& Lovelace, 1983; Potts, 1974) used in previous research. Nevertheless, the analogy will be a useful one if it provides new insight into macrospatial cognitive processes.

In this regard, recent research on categorization effects with unidimensional stimulus continua, particularly with symbolic spatial linear orders (Maki, 1981), indicates a new approach to studying the phenomenon of route segmentation (Downs \& Stea, 1973). It has been suggested that for the sake of cognitive economy, the continuous flow of temporospatial experience during route learning is organized into "chunks" or segments. This organizational process may be based on topological properties of the environment (e.g., similarity, enclosure, or "belongingness") or on a macrospatial convention (e.g., measuring urban space in terms of city blocks).

Route segmentation is conceptually similar to categorization along symbolic spatial linear orders. An example of the latter is provided in Maki's (1981) studies of comparative judgments along an East-West dimension involving a set of cities (stimuli) in two adjacent states (categories). It was found that response latencies for judgments concerning two cities within the same state were inversely, but directly, related to intercity distance, whereas latencies for similar judgments concerning two cities in different states were not systematically related to intercity distance. Extrapolating freely from these findings to the issue of route segmentation, it would be predicted that distance judgments involving locations within the same segment would be more directly affected 
by the physical distance between the locations involved than would similar judgments involving two locations in adjacent segments.

Allen (1981) found evidence of segmentation effects in a study in which children and adults were instructed to make two-choice proximity judgments among features along a route learned from a slide presentation. Adults were able to make accurate decisions regarding which of two comparison scenes was nearer a reference scene when all three scenes were within a common route segment, but they grossly distorted their estimates of proximity when the nearer comparison scene was in a route segment adjacent to the one in which the reference scene and more distant comparison scene were located. In such cases, the comparison scene within the same segment was reliably judged to be closer to the reference point, even though it was up to three times the distance from the reference point to the comparison scene in the adjacent route segment.

On the basis of these findings, Allen suggested that distance judgments between points in different route segments involve a distance-insensitive/segment-inclusion process that is a form of categorization, whereas distance judgments within segments require a distance-sensitive computation and comparison process. This explanation is in need of support from additional evidence, including a replication and extension of previous findings. A replication would be desirable simply to substantiate previous results, particularly the extreme distortion characterizing intersegment judgments. An extension would be necessary to demonstrate that the two-alternative proximity judgment task used in Allen (1981) did not in itself predispose subjects toward relying on route segmentation. Specifically, it would be useful to demonstrate that route segmentation influences performance in a distance-estimation task, which clearly orients subjects toward some form of distance computation. Also, it would be important to demonstrate that the influence of route segmentation is a rather pervasive phenomenon, detectable in even a straightforward distance-estimation task. Such a demonstration would be significant because of the widespread use of distance-estimation tasks in the study of spatial cognition (Briggs, 1976).

The present series of studies was designed in response to the need for additional research on these issues. Specifically, Experiments 1 and 2 were designed to provide evidence of the replicability of Allen's (1981) results. In addition, Experiment 2 provided a comparison of the effects of route segmentation on proximity judgments and distance estimates. In Experiment 3, estimates of subjects in a unidirectional distance-estimation task were examined for evidence of the influence of route segmentation.

\section{EXPERIMENT 1}

The route selected for the studies in this series was heterogeneous in that it proceeded through a variety of ur- ban terrain. The purpose of Experiment 1 was to identify segments within this route by having subjects designate segment boundaries. These boundaries were essential for the construction of distance-judgment problems that would reveal the influence of route segmentation on subjects' conceptions of macrospatial distance.

\section{Method}

Subjects. Data were collected from 25 females and 25 males of college age who voluntarily participated in the study for research credit in introductory psychology classes. None of the subjects was familiar with the route depicted in the slide presentation.

Materials. A slide presentation portraying a 1,000 -m walk was constructed of 60 color slides with a standard $20-\mathrm{m}$ interval between successive scenes. The only exceptions to this interval occurred during extreme changes in heading (e.g., turning a corner) in which case perceptual continuity was preserved by providing $50 \%$ visual overlap in successive slides. The course of the route, as shown in Figure 1, continued through a park, a college campus, and several blocks of a residential neighborhood. During the presentation, slides were projected automatically at a rate of $5 \mathrm{sec} / \mathrm{scene}$ using a Kodak Ektagraphic slide projector.

Procedure. The procedure involved a viewing phase and a testing phase. In the viewing phase, subjects viewed the slide presentation twice. After each view, subjects were presented with a task that directed their attention to the order in which scenes appeared during the presentation. This task required subjects to designate the correct order in which five scenes from the route appeared; the scenes (color prints made from slides) were from the beginning of the route, one-quarter of the way through the route, one-half of the way through the route, three-quarters of the way through the route, and the end of the route. All subjects ordered the scenes correctly after two views of the route. In the testing phase, subjects were instructed to go through the slide series a third time, designating different parts of the route. They were given a remote control unit to the slide projector and asked to proceed through the walk at their own pace. Each time they began what appeared to them to be a new part of the walk, they were to inform the experimenter, who recorded the ordinal positions of the slides involved in the boundary. No minimum or maximum was placed on the number of parts the route could contain.

\section{Results}

The proportion of subjects selecting each slide in the series as the first scene in a new part of the walk is shown in Figure 2. Subjects made 270 such designations, with a mean of 5.4, a mode of 5.0, a median of 5.0, and a standard deviation of 1.56 . It was inferred on the basis of these results that the route could legitimately be divided by five boundaries into six distinct segments. These boundaries were based on environmental features pictured in the slides, not on the slides themselves. Generally, specific features were prominent visually in three successive slides (covering $60 \mathrm{~m}$ ). To accommodate this perceptual overlap, boundaries were marked by triads of consecutive scenes rather than by individual scenes. The five triads that included the scenes selected by the highest cumulative proportion of subjects (i.e., the sum of subjects selecting a scene in the triad divided by total number of subjects) were used to determine the locations of the boundaries within the context of the route (see Figure 2).

For the purpose of estimating the proportion of subjects that would have selected scenes within each of these 


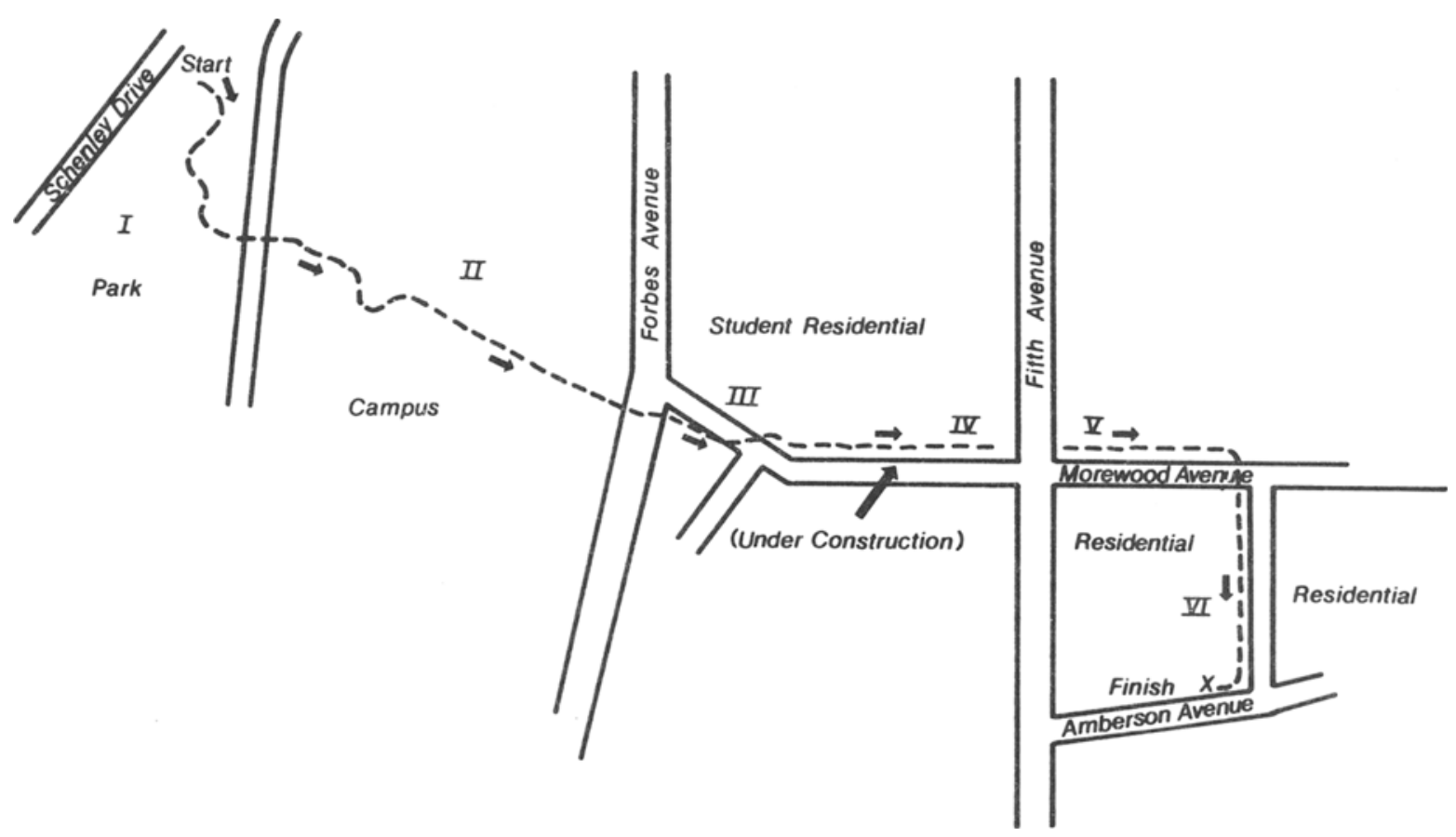

Figure 1. Course of the walk depicted in the slide presentation.

established boundaries on the basis of chance, it was assumed that each subject selected a total of five scenes and that selections were independent events. Under these assumptions, the proportion of subjects selecting a scene within a boundary triad on the basis of chance was 25 . When an approximation to the $\mathrm{z}$ distribution was used to compare this chance level with observed performance, it was found that the proportion of subjects selecting scenes constituting one of the five boundary triads exceeded that expected by chance (all zs $>3.20$ ). No other triad of scenes exceeded chance level (all zs $<1.12$ ).

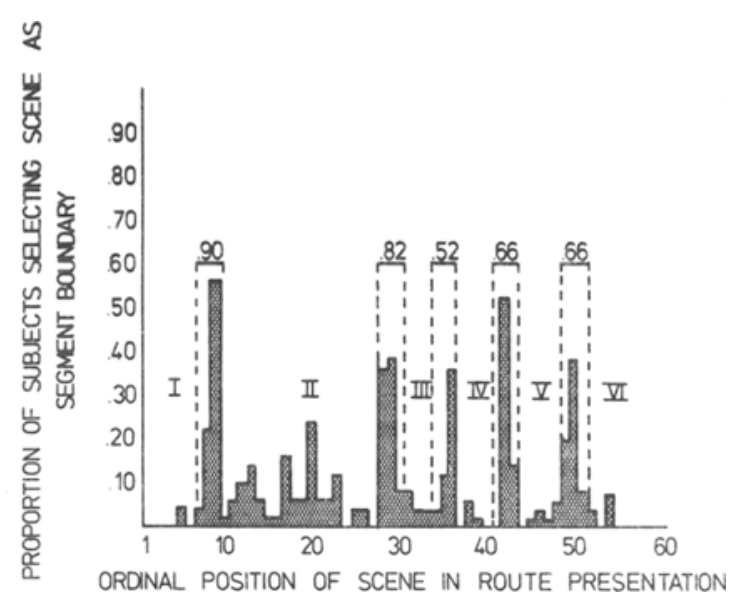

Figure 2. Triads of scenes selected as boundaries between route segments.

\section{Discussion}

These results indicated that the route shown in the slide presentation consisted of six segments, including a wooded park (I), a college campus (II), a block of fraternity and dormitory housing (III), a street under construction (IV), and two blocks of large single-family dwellings ( $V$ and VI). Segments I and II were separated by a small parking lot, segments II and III by a heavily traveled street, segments III and IV by a change in the type of buildings along the street and by the beginning of street construction, segments IV and V by another major thoroughfare, and segments $\mathrm{V}$ and VI by a $90^{\circ}$ turn to the right from an area with houses on one side of the street to a block with houses on both sides of the street.

These findings provide some support for the proposition that route information is partitioned into segments during the course of normal macrospatial experience. Of course, the fact that subjects were able to comply with the instructions to designate various parts of the walk cannot be taken to mean that the resulting segments have some influence on judgments of macrospatial distance. However, the segments specified in this study were the same as those discussed in this context in Experiment 2.

It must be acknowledged that there is no wellestablished means for determining the boundaries between route segments for group data. The technique employed in this study was selected because it reflected group consensus regarding the number of segments and their locations. Although a slightly different technique that did not limit the designation of boundaries to three consecutive slides had been used in a previous study (Allen, 1981), 
the present technique, which defined boundaries more conservatively, is preferable. When the extent of boundaries is not limited, the resulting boundaries could cover a larger geographic area than the route segments they separate. Not only would this be unsound conceptually, but it would limit the validity of studies (such as Experiment 2) designed to demonstrate the effect of route segmentation on distance estimates.

\section{EXPERIMENT 2}

The purpose of this study was to determine the influence of route segmentation on judgments of proximity among various locations along a route. It was predicted that proximity judgments involving locations within the same segment would be more influenced by metric distance than would proximity judgments involving locations in adjacent segments. To test this prediction, six different types of proximity problems were constructed using the route segments identified in Experiment 1 (see Table 1). All problems involved a judgment of relative proximity, that is, a decision with regard to which of two comparison scenes (locations) was nearer a reference scene (location). In all cases, one comparison scene specified a viewpoint prior to the reference scene and the other specified a viewpoint after the reference scene along the course of the route.

In intersegment problems, all three scenes were situated within the same route segment. An example using the route segments identified in Experiment 1 would be a reference scene and two comparison scenes from the college campus segment of the pictorialized route. In contradictory intersegment problems, the comparison scene that was closer to the reference scene was located in a segment adjacent to the one in which both the reference scene and the other comparison scene were situated. These are referred to as "contradictory" because reliance on segment information leads to a proximity decision that is different from that made on the basis of metric distance information. An example would be a location in the park segment of the route that was nearer a reference location in the campus segment than was a second location within the campus segment. In such cases, reliance on segmentation information alone leads to a metrically inaccurate judgment. In noncontradictory intersegment problems, a comparison scene was nearer a reference scene within the same route segment than was a second comparison scene in an adjacent segment. An example would be a location within the park segment of the route that was closer to a reference location within the park segment than was a second location in the campus segment of the route. In such cases, reliance on segmentation information leads to a metrically accurate judgment.

In these problems, metric accuracy of the proximity judgment, that is, selecting the comparison scene that actually is nearer the reference scene, can serve as an indicator of the influence of metric distance. According to predictions derived from other studies of judgments along spatial linear orders, metric distance should influence intrasegment judgments to a greater extent than contradictory and noncontradictory intersegment judgments. Thus, intrasegment judgments would be expected to result in greater accuracy than would contradictory intersegment judgments. However, noncontradictory judgments would also be expected to be very accurate because a metrically accurate decision could be reached on the basis of either segmentation information or metric distance information. For this reason, difficulty of distance discrimination was introduced as another factor useful in detecting the influence of route segmentation on proximity judgments. Difficulty of distance discrimination was varied by manipulating the ratio between the shorter distance (i.e., from the reference scene to the nearer comparison scene) and the longer distance (i.e., from the reference scene to the more distant comparison scene) in the problem. Two levels of difficulty were included for each of the three problem types described previously; these will be referred to as easy discriminations ( $1: 3$ ratio) and difficult discriminations (1:1.5 ratio).

If subjects' judgments were strongly influenced by metric distance, then accuracy should be greater for easy discriminations than for difficult discriminations. This seemingly sensible contingency described expectations for intrasegment judgments. However, because intersegment judgments were expected to be little influenced by metric distance, accuracy for these problem types was not expected to vary as a function of difficulty of distance discrimination. In other words, $1: 3$ and 1:1.5 distance ratios were expected to yield equally accurate judgments in the case of contradictory and noncontradictory intersegment problems.

Overall predictions for the various types of problems may be summarized as follows:

(1) Accuracy for intrasegment judgments was expected

Table 1

Six Types of Distance Judgment Problems for Experiment 2

\begin{tabular}{lcc}
\hline \multicolumn{1}{c}{ Segmentation Condition } & Distance Ratio & $\begin{array}{c}\text { Predicted Probability of } \\
\text { Correct Response }\end{array}$ \\
\hline Intrasegment & $1: 3$ & $\mathrm{p}>.50$ \\
Intrasegment & $1: 1.5$ & $\mathrm{p} \geq .50$ \\
Contradictory Intersegment & $1: 3$ & $\mathrm{p}<.50$ \\
Contradictory Intersegment & $1: 1.5$ & $\mathrm{p}<.50$ \\
Noncontradictory Intersegment & $1: 3$ & $\mathrm{p}>.50$ \\
Noncontradictory Intersegment & $1: 1.5$ & $\mathrm{p}>.50$ \\
\hline
\end{tabular}


to be no worse than chance level ( .50 for a two-alternative choice) and higher for easy discriminations than for difficult discriminations. This prediction was based on the contention that subjects would calculate and compare metric distances for these problems.

(2) Accuracy for contradictory intersegment judgments was expected to be below chance level, with no difference between easy discriminations and difficult discriminations. This prediction was based on the contention that subjects would rely strictly on segmentation information for these problems.

(3) Accuracy for noncontradictory intersegment judgments was expected to be above chance level, with no difference between easy discriminations and difficult discriminations. This prediction was also based on the contention that subjects would rely strictly on segmentation information for these problems.

Two different tasks involving the six problem types in Table 1 were included in the present study. In the twoalternative proximity-judgment task, subjects indicated which of two comparison scenes was nearer a reference scene, as indicated previously. In the distance-estimation task, subjects estimated distance between the reference scenes and individual comparison scenes. The same set of reference and comparison scenes was used in both tasks. The purpose of including the distance estimation task was to insure that evidence of the influence of route segmentation on distance judgments would not be considered an artifact of the forced-choice procedure in the two-alternative proximity judgment task.

\section{Method}

Subjects. Data were collected from 20 males and 20 females of college age who voluntarily participated in the study for research credit in introductory psychology classes. None of the subjects was familiar with the route depicted in the slide presentation.

Materials. The slide presentation used in this study was the same as that described in Experiment 1. All scenes used in the distancejudgment tasks were $8.8 \times 12.7 \mathrm{~cm}$ color prints made from slides composing the presentation. For the two-alternative proximityjudgment task, pairs of comparison scenes were mounted side by side on a single card and the reference cards were mounted individually. All scenes involved in the distance-estimation task were mounted on small individual cards. Scenes from the route were selected for the construction of four examples of each of the six problem types described in Table 1 . The result was 24 problems, each consisting of a reference scene and a pair of comparison scenes. For all pairs of comparison scenes, the scene mounted on the left appeared before the reference scene and the scene mounted on the right appeared after the reference scene along the route. The direction of the nearer of the two comparison scenes to the reference scene was counterbalanced so that the metrically correct choice appeared before and after the reference scene an equal number of times. For convenience, the same scene served as a reference scene for two different problems.

During the proximity judgment and estimation tasks, scenes were presented on a two-tiered stand. The reference scene was placed on the upper level of the stand, and the comparison scene(s) was (were) placed on the lower level. Two horizontally arranged buttons, one under each comparison scene, were attached to the test stand facing the subject. Depression of either button activated a corresponding signal light on the side of the test stand facing the experimenter. The buttons were not used in the estimation task.
Procedure. The procedure involved a viewing phase and a testing phase. During the viewing phase, subjects viewed the slide presentation twice. Scenes were presented at a rate of $5 \mathrm{sec} / \mathrm{slide}$. After each view of the route, subjects were given five scenes (color photographs) from the walk and instructed to order them in the sequence in which they appeared during the presentation (see Experiment 1). All subjects ordered the five scenes correctly after two views of the route. During the testing phase, each subject performed both the two-alternative proximity-judgment task and the distanceestimation task. Order of tasks was counterbalanced. The proximityjudgment task involved 24 trials (four examples of the six problem types described above). Two practice trials, during which the task was thoroughly explained in the context of two sample problems, were provided prior to data collection. During testing, the experimenter placed the reference scene on the upper level of the test stand and the pair of comparison scenes on the lower level approximately $4 \mathrm{sec}$ later. Subjects then responded by pressing the button beneath the comparison scene that was nearer the reference scene in terms of walking distance. It was made clear to the subjects that the precise geographic locations to be considered in making the proximity judgments were specified by the environmental features visible on the lower border of each picture. This convention was chosen instead of one that would have had subjects imagine the geographic points from which test scenes were photographed. The 24 problems were presented in the same random order to all subjects.

The distance-estimation task involved 48 trials. In these trials, the individual reference and comparison scenes were the same as those used in the proximity-judgment task. Two practice trials, during which the magnitude-estimation procedure was thoroughly explained within the context of two sample estimates, were provided prior to data collection. No standard stimulus value was presented in the magnitude-estimation procedure; subjects devised their own scales. During testing, the experimenter placed the reference scene on the upper level of the test stand and the comparison scene on the lower level approximately $4 \mathrm{sec}$ later. Subjects responded by providing a verbal estimate of the distance between the reference scene and the comparison scene. It was made clear to the subjects that the precise geographic points to be considered in making estimates were specified by environmental features visible on the lower border of each photograph. The 48 distance-estimation trials were presented in the same random order to all subjects.

\section{Results}

The dependent variable of primary interest was the proportion of each type of problem (number correct out of four possible) in which a metrically correct judgment was made. These proportions were easily computed for the data from the two-alternative proximity-judgment task. For the data from the distance-estimation task, these proportions were computed by "reassembling" the twoalternative format, that is, by comparing the two estimates of distance (between reference scene and comparison scene) in each two-alternative problem. Thus, a metrically correct judgment was one in which the estimate from the reference scene to the nearer comparison scene was lower than the estimate from the same reference scene to the more distant comparison scene. The resulting proportion scores were analyzed by comparing them against chance level (.50) and by comparing them to each other in an analysis of variance.

The data from the distance-estimation task provided judged-distance ratio as another dependent variable of interest. Judged-distance ratio was computed by determining the average ratio of the lesser distance to the greater 
distance for each problem type. For these ratios, the lesser distance was given a value of 1 , so that, for example, estimates of 20 and 45 yielded a ratio of 1:2.25. Ratios were converted to proportions (e.g., 1:2.25 $=.44$ ) before being analyzed.

As another means of contrasting the role of metric distance in the three types of problems, individual subjects' distance estimates were analyzed to provide power functions of actual distance. The exponents, intercepts, and correlation coefficients from these functions were subjected to ANOVAs.

Comparisons to chance-level performance. The mean proportion of metrically correct responses, broken down according to problem type and task, is presented in Table 2 . These accuracy levels were compared to .50 using an approximation to the normal distribution. As predicted, accuracy was significantly above .50 for $1: 3$ intrasegment problems and for both $1: 3$ and $1: 1.5$ noncontradictory intersegment problems; not significantly different from .50 for 1:1.5 intrasegment problems; and significantly below .50 for both $1: 3$ and $1: 1.5$ contradictory intersegment problems. This pattern held for comparisons resulting from both the two-alternative proximity-judgment task and the distance-estimation task, with the single exception of accuracy for contradictory intersegment problems with a 1:3 distance ratio. For these problems in the distanceestimation task, accuracy was not quite significantly below .50 $(\mathrm{z}=-1.53)$.

Analysis of proportion correct. The mean proportion of metrically correct judgments was subjected to an arcsine transformation and then analyzed in a 2 (order of task presentation) $\times 2$ (task: proximity judgment vs. distance estimation) $\times 6$ (problem type) mixed ANOVA. This analysis yielded a significant effect for problem type $[F(5,190)=118.70, \mathrm{MSe}=.39]$. (The $\mathrm{p}<.05$ criterion was used throughout these experiments.) No other main effects or interactions were significant. Scheffé comparisons revealed four different levels of accuracy among the six problem types. The lowest level was for $1: 3$ and $1: 1.5$ contradictory intersegment problems, which did not differ from each other in accuracy. Significantly above these were 1:1.5 intrasegment problems, which in turn were significantly lower than 1:3 intrasegment problems in accuracy. The highest accuracy levels were for $1: 3$ and 1:1.5 noncontradictory intersegment problems, which did not differ in accuracy.

Table 2

Mean Proportion of Metrically Correct Judgments

\begin{tabular}{llllc}
\hline \multicolumn{2}{c}{ Type of Problem } & & & \multicolumn{2}{c}{ Type of Task } \\
\cline { 1 - 2 } \cline { 5 - 5 } Segmentation Condition & $\begin{array}{c}\text { Distance } \\
\text { Ratio }\end{array}$ & $\begin{array}{c}\text { Proximity } \\
\text { Judgment }\end{array}$ & $\begin{array}{c}\text { Distance } \\
\text { Estimation }\end{array}$ \\
\hline Intrasegment & $1: 3$ & & $.719^{*}$ & $.713^{*}$ \\
Intrasegment & $1: 1.5$ & & .575 & .594 \\
Contradictory Intersegment & $1: 3$ & & $.256^{* *}$ & .331 \\
Contradictory Intersegment & $1: 1.5$ & & $.238^{* *}$ & $.281^{* *}$ \\
Noncontradictory Intersegment & $1: 3$ & & $.863^{*}$ & $.888^{*}$ \\
Noncontradictory Intersegment & $1: 1.5$ & $.881^{*}$ & $.881^{*}$ \\
\hline
\end{tabular}

*Significantly above .50. **Significantly below .50 .
Analysis of judged-distance ratios. The pairs of distance estimates corresponding to the 24 two-alternative proximity-judgment problems were made into ratios and compared with their corresponding veridical distance ratios (either $1: 3$ or $1: 1.5$ ). These comparisons involved converting the ratios to proportions and using an approximation to the normal distribution. For intrasegment problems, actual ratios of $1: 3$ and 1:1.5 were estimated to be $1: 1.85$ and $1: 1.47$, respectively. For contradictory intersegment problems, actual ratios of $1: 3$ and $1: 1.5$ were estimated to be $1: 0.72$ and 1:0.63, respectively. Note that these two ratios indicate that the shorter distances were in fact estimated to be greater than the longer distances. For noncontradictory intersegment problems, actual ratios of $1: 3$ and $1: 1.5$ were estimated to be $1: 2.60$ and $1: 2.27$, respectively.

Only those averages for the $1: 1.5$ intrasegment problems (ratio $=1: 1.47$ ) and the $1: 3$ noncontradictory intersegment problems (ratio $=1: 2.6$ ) did not differ significantly from the actual distance ratios. Longer distances were underestimated proportionately for $1: 3$ intrasegment problems and for both $1: 3$ and $1: 1.5$ contradictory intersegment problems. Longer distances were overestimated proportionately for $1: 1.5$ noncontradictory intersegment problems.

Analyses of power-function parameters. Individual subjects' distance estimates were analyzed in a series of linear regression analyses in which log estimates were compared to log distances for intrasegment judgments, contradictory intersegment judgments, and noncontradictory intersegment judgments. These analyses yielded three parameters of interest: exponent of the power function (reflecting the relationship between estimated and actual distance), intercept of the power function, and correlation coefficient (reflecting the linearity of the function). Correlation coefficients were transformed $r$ to $z$ for analyses. Separate one-way ANOVAs were performed on these parameters, with type of judgment serving as the independent variable. Each ANOVA revealed a significant effect of type of judgment [all $\mathrm{Fs}(2,78)>4.50$ ]. Scheffé comparisons were used to delineate differences among the three judgment types. The mean exponen derived from intrasegment functions (.92) was significantly greater than that derived from noncontradictory intersegment functions (.48), which in turn was greater than that derived from contradictory intersegment functions $(-.02)$. The results for the intercept were the inverse of those for the exponent; mean intercept was greater for contradictory intersegment functions (1.54) than that for noncontradictory intersegment functions (.45), which was in turn greater than that for intrasegment functions (.03). Mean correlations (transformed back from $\mathrm{z}$ ) derived from intrasegment (.76) and noncontradictory intersegment (.57) functions did not differ from each other, but both were greater than the correlation derived from contradictory intersegment functions $(-.05)$. The mean exponent and mean correlation coefficient from the contradictory intersegment functions suggest an essentially flat function 
with a lack of systematicness. The mean intercept for this type of function, considered with the exponent, simply indicates an overestimation of short distances and an underestimation of long distances.

\section{Discussion}

The preceding results generally confirm the predictions for the six types of problems and provide strong evidence that route segmentation affects judgments of macrospatial distance. Accuracy for intrasegment judgments was no worse than chance level $(.50)$ and was greater for easy (1:3) discrimination than for difficult (1:1.5) discriminations; accuracy was below chance level for contradictory intersegment judgments, with no difference between easy and difficult discriminations; and accuracy was above chance level for noncontradictory intersegment judgments, with no difference between easy and difficult discriminations. The fact that the same accuracy pattern (with the one minor exception noted previously) was found for comparisons based on proximity judgments and for those based on distance estimates indicates that these findings were not an artifact of a particular procedure.

Further illustration of the biasing effects of route segmentation was provided by the analyses of parameters from subjects' estimated-to-actual distance power functions. These analyses indicated a very strong positive relationship between estimated and actual distance in the case of intrasegment estimates, a moderate positive relationship in the case of noncontradictory intersegment estimates, and virtually no relationship in the case of contradictory intersegment estimates.

\section{EXPERIMENT 3}

The results of Experiment 2 indicated that the influences of the cognitive organization of route knowledge were not limited to two-alternative proximity judgment tasks. The purpose of Experiment 3 was to seek evidence that these influences extended to simple unidirectional distance estimates made across multiple segment boundaries. If route segmentation exerted strong effects under such circumstances, estimates from a standard reference point at the beginning of the route to a set of locations within a common route segment would be expected to have a limited range and to increase very little as a function of distance increments. In contrast, estimates to a set of locations that crossed a segment boundary would be expected to have a relatively large range and to increase systematically. In other words, estimates should be a "stairstep" function of actual distance. An orderly relationship between estimated and actual distance should hold for the route as a whole (as a result of route segments being ordered in terms of increasing distance). However, this relationship would be primarily due to across-boundary increments in estimated distance rather than to within-segment increments.

Distance-estimation data were examined in two ways for effects of route segmentation in the present experiment. First, raw estimates were analyzed for evidence of (a) an overall increase in estimated distance over the route as a whole and (b) the presence or absence of significant differences among estimates to locations within the same segment. An overall significant increase in estimates over the route was expected, but significant increases in estimated distance would not appear within a segment if the proposed "stairstep" function were obtained.

In addition, subjects' estimates to sets of locations within a common segment and their estimates to sets of locations crossing segment boundaries were converted to power functions (log estimates as a function of $\log$ distances). Exponents, intercepts, and correlation coefficients from these functions were compared using $t$ tests. If the proposed "stairstep" function were present in the estimation data, the mean exponent for the across-boundary functions would be greater than that for the within-segment functions, and the latter should approximate a value of .00 . If the inverse relationship between exponent and intercept suggested in Experiment 2 held, mean intercept for within-segment functions should be greater than that for across-boundary functions. Also based on the results of Experiment 2, the mean correlation coefficient should be greater for across-boundary functions than for withinsegment functions.

It is useful to point out that all of the estimates under discussion are intersegment judgments, that is, they represent judgments of the distance from a standard reference point at the beginning of the route to 24 target locations along the way. The term "across-boundary" is used to denote a set of target locations that includes locations in two adjacent segments, and the term "common segment" is used to denote a set of target locations confined to a single segment of the route.

\section{Method}

Subjects. Data were collected from ten males and ten females of college age who participated voluntarily for research credit in introductory psychology courses. None of the subjects was familiar with the route depicted in the slide presentation.

Materials. The slide presentation used in this study was the same as that described in Experiment 1. Twenty-four slides from the presentation were duplicated for use as target scenes in the distanceestimation task. These included four scenes from each route segment identified in Experiment 1, two scenes randomly selected from the first half and two from the last half of each segment. A Kodak Ektagraphic slide projector was used to present the route and target scenes. Subjects provided written estimates on a numbered response sheet.

Procedure. Subjects viewed the slide series twice and completed the distance-estimation procedure after each presentation. The initial presentation and estimation experience served to familiarize the subjects with the route and the procedure. As in Experiments 1 and 2 , the data of interest were those obtained after two viewings of the route. During the presentation, slides were projected automatically at a rate of $5 \mathrm{sec} / \mathrm{slide}$. At the conclusion of each presentation, subjects were provided response sheets and were instructed in the magnitude-estimation procedure for estimating distances from the beginning of the route to 24 other locations along the route. Practice trials were given to ensure that instructions had been understood. No standard stimulus value was presented; subjects developed their own ratio scales of distance. It was made clear that the precise geographic locations to be considered in making distance 
estimates were specified by the environmental features visible on the lower border of the slides. The 24 target slides were presented in the same random order to all subjects. Subjects were tested in groups of ten.

\section{Results}

Analysis of estimates. Subjects' estimates were subjected to a one-way ANOVA with target scenes as the independent variable. This analysis revealed a significant increase in estimates across target scenes $[\mathrm{F}(23,437)=$ $9.55, \mathrm{MSe}=3396.83]$. The specific hypothesis underlying this study concerned differences among estimates made to target scenes within the same route segment. Accordingly, a series of planned comparisons involving the mean estirnates for locations within the same route segment were conducted. The mean estimates used in this procedure are shown in Figure 3. The statistically significant critical difference was 48.65 ; according to this criterion, there were no significantly different means within segments.

Analyses of power-function parameters. All 24 distance estimates-to four locations in each of six segments-were made from a standard reference point at the route's origin. Estimates from the reference point to the four locations in the first route segment were intrasegment judgments (i.e., the reference point and all estimates involved the same segment). Accordingly, they were considered qualitatively different from the others and were excluded from analyses. This left a total of five sets of estimates to locations in a common segment. Individual subjects' log estimates to these locations were analyzed in linear regression analyses that yielded power functions for each route segment. For each subject, a mean exponent, intercept, and correlation coefficient were obtained from these functions. Means for these parameters were obtained in an effort to counteract the potential instability of these functions resulting from restriction of range. Correlation coefficients were converted to $\mathrm{z}$ scores for determining means and for inclusion in all subsequent analyses.

Power functions for across-boundary estimates were ob-

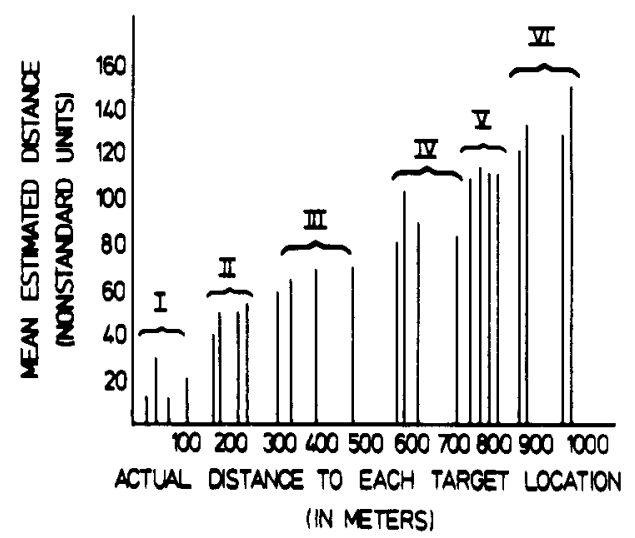

Figure 3. Mean estimates to 24 target locations (four targets in each of six route segments). tained using subjects' log estimates to the two target locations in each segment immediately preceding a boundary and the two target locations immediately following that boundary. The five boundaries thus provided five sets of these estimates, with four locations involved in each. For each subject, a mean exponent, intercept, and correlation coefficient were obtained from these functions.

Because restriction of range was a concern, the actual distances involved in within-segment functions and across-boundary functions were matched as closely as possible. Within-segment distances covered a range of 60 to $160 \mathrm{~m}$; across-boundary functions covered a range of 60 to $200 \mathrm{~m}$. The range of distances involved in the route as a whole was $960 \mathrm{~m}$.

Separate $t$ tests for related measures were performed on mean exponents, intercepts, and correlation coefficients from the within-segment and across-boundary functions. All three analyses revealed significant differences [all $\operatorname{ts}(19)>2.15]$. The mean exponent for across-boundary functions was greater than that for within-segment functions ( .80 vs. -.02$)$; mean intercept value was larger for the within-segment functions than for across-boundary functions ( 2.06 vs. .44); and mean correlation coefficient (converted from $\mathrm{z}$ ) was larger for across-boundary functions than for within-segment functions (.46 vs. .33).

An additional groupwise regression analysis was performed to obtain a power function describing the relationship between estimated and actual distance over the route as a whole. This analysis yielded the function $y=$ $-.49 x^{80}(r=.98)$. This function indicates a strong, orderly, positive relationship between estimated and actual distance when all the data are analyzed together. This function approximates the $y=3.82 x^{.74}(r=.98)$, obtained by Allen et al. (1978), suggesting that the estimates in the present study are not atypical.

\section{Discussion}

The results of Experiment 3 suggest that route segmentation has a noticeable effect on judgments of distances along a route in a simple distance estimation task in which a series of estimates are made from a single reference point at the beginning of the route. This inference is based on (1) the absence of statistically significant differences among estimates to locations within the same route segment, (2) significantly greater exponents from functions describing across-boundary estimates than from functions describing estimates to locations within the same segment, and (3) an exponent approximating .00 for the function describing estimates to locations within the same segment. The results must be interpreted in the context of a significant increase in estimates over the route as a whole.

The first of these findings has little impact by itself because it is a negative result. However, the remaining results provide rather strong support for the hypothesis that route segmentation affects performance in a straightforward distance-estimation task. In particular, it supports the proposed "stairstep" relationship between estimated and actual distance. It is not unreasonable to suggest that 
route segmentation may have had a subtle effect on the results of previous studies in which distance estimation procedures were used to examine macrospatial knowledge.

\section{GENERAL DISCUSSION}

The results of Experiments 1, 2, and 3 support the propositions that individuals tend to organize their experience into distinct segments during the acquisition of route knowledge and that these segments significantly influence subsequent judgments of macrospatial distances. It was demonstrated in Experiment 1 that subjects were capable of dividing an actual route into segments in a laboratory setting. There was considerable agreement as to the boundaries separating these segments. In Experiment 2 , it was shown that the segments exerted a powerful influence on subjects' distance judgments, both in a proximity-judgment task and in a distance-estimation task. Specifically, the evidence indicated that intrasegment judgments were much more affected by metric distance than were intersegment judgments. The findings of Experiment 3 suggested that route segmentation also influenced distance estimates in a simple task in which unidirectional distances were estimated from a point of origin at the beginning of a route. Specifically, it was shown that although estimates tended to increase as a function of distance for estimates over the route as a whole and for estimates across individual segment boundaries, estimates to locations within the same segment generally did not increase systematically.

From the perspective of macrospatial cognition, the present research provides insight into the organization of route representations and the process involved in making estimates of macrospatial distances. Evidence suggests that route segmentation as an analog of categorization is responsible for systematic distortion of distance judgments, including estimates of metric distance. Presumably, these effects would subside somewhat as individuals become more familiar with the route. However, studies by Byrne (1979), Lynch (1960), and Stevens and Coupe (1978) indicate that some distortions of geographic relationships are a reliable feature in the macrospatial knowledge of long-term residents in the area. Perhaps it is the case that route segmentation is initially a means of facilitating learning, but that over the course of repeated encounters many segments are integrated according to a variety of functional or practical criteria. The result would be a developmental trend toward relatively larger, more stable segments. This and other possibilities should be explored in future research.

From a broader viewpoint, these studies afford an empirical link between macrospatial cognition as a specialized research area and the study of human cognition in general. The findings of Experiment 2, in particular, suggest a similarity or analogy between route segmentation on the one hand and categorization along linear dimensions on the other. Previous research has found intra- category response latencies-but not intercategory latencies-for comparative distance judgments along linear spatial orderings to be directly related to interstimulus distances. The present study provided evidence that intrasegment distance judgments--but not intersegment judgments-are directly related to metric interlocation distance. It is interesting to speculate that this similarity represents a homology rather than an analogy. The phenomenon of route segmentation in the early cognitive history of the human species (or of the individual) may have provided the basis for the later phenomenon of categorization in symbolic linear order representations. This line of speculation parallels that of Holyoak and Mah (1982) in their discussion of the ecological basis of the biasing effects of cognitive reference points on distance judgments along symbolic spatial dimensions.

Nevertheless, it should be emphasized that the conclusions drawn from the preceding studies are subject to two qualifications originally pointed out by Wilton (1979). First, route segmentation is subtly different from categorization as it is often defined (e.g., Rosch \& Lloyd, 1978). Questions regarding boundaries and defining characteristics can be answered only roughly at this point, and the explanation of a hierarchy of spatial elements is not absolutely necessary to account for the result obtained. Second, no assumptions are made with regard to whether route representations (which are posited as being involved directly in the products of distance estimates) should be considered as continuous or discrete arrays. The present evidence can be used only to argue for different levels of discrimination among spatial locations. Nevertheless, these two qualifications do not detract from the major contributions of this research, namely (1) the delineation of different modes or levels for representing spatial information in linear order format, and (2) the demonstration of the robust effects of this phenomenon on judgments of macrospatial distances.

\section{REFERENCES}

Allen, G. L. (1981). A developmental perspective on the effects of "subdividing" macrospatial experience. Joumal of Experimental Psychology: Human Learning and Memory, 7, 120-132.

Allen, G. L., Siegel, A. W., \& Rosinski, R. R. (1978). The role of perceptual context in structuring spatial knowledge. Journal of Experimental Psychology: Human Learning and Memory, 4, 617-630.

BECK, R. J., \& WOOD, D. (1976). Cognitive transformation from urban geographic fields to mental maps. Environment and Behavior, 8, 199-238

Briggs, R. (1976). Methodologies for the measurement of cognitive distance. In G. Moore \& R. Golledge (Eds.), Environmental knowing (pp. 325-334). Stroudsburg, PA: Dowden, Hutchinson, \& Ross.

BYRNE, R. W. (1979). Memory for urban geography. Quarterly Journal of Experimental Psychology, 31, 147-154

Downs, R. M., \& STEA, D. (1973). Cognitive maps and spatial behavior: Products and process. In R. Downs \& D. Stea (Eds.), Image and environment (pp. 8-26). Chicago: Aldine.

FiShBEIN, H. D. (1976). Evolution, development, and children's learning. Pacific Palisades, CA: Goodyear.

Hazen, N. L., Lockman, J. J., \& Pick, H. L. (1978). The development of children's representations of large-scale environments. Child Development, 49, 623-636. 
HolyoAK, K. J. (1978). Comparative judgments with numerical reference points. Cognitive Psychology, 10, 203-243.

Holyoak, K. J., \& MAH, W. A. (1982). Cognitive reference points in judgments of symbolic magnitude. Cognitive Psychology, 14, 328-352.

Klahr, D., Chase, W. G., \& Lovelace, E. A. (1983). Structure and process in alphabetic retrieval. Journal of Experimental Psychology: Learning, Memory, and Cognition, 9, 462-477.

Kosslyn, S. M., Murphy, G. L., Bemesderfer, M. E., \& Feinstein, K. J. (1977). Category and continuum in mental comparisons. Journal of Experimental Psychology: General, 106, 341-376.

Kuipers, B. J. (1978). Modeling spatial knowledge. Cognitive Science, 2, 129-153

LYNCH, K. (1960). The image of the city. Cambridge: MIT Press.

MAKI, R. H. (1981). Categorization and distance effects with spatial linear orders. Joumal of Experimental Psychology: Human Learning and Memory, 7, 15-32.

Moar, I., \& CARLeton, L. R. (1982). Memory for routes. Quarterly Journal of Experimental Psychology, 34A, 381-394.

Moyer, R. S., \& Dumais, S. T. (1978). Mental comparison. In G. H. Bower (Ed.), The psychology of learning and motivation (Vol. 12 , pp. 117-156). New York: Academic Press.

Newell, A., \& Simon, H. A. (1972). Human problem solving. Englewood Cliffs, NJ: Prentice-Hall.

Norman, D. A., Rumelhart, D. E., \& The LNR Research Group. (1975). Explorations in cognition. San Francisco: Freeman.

Piaget, J., \& INHeLder, B. (1967). The child's conception of space. New York: Norton.
Piaget, J., Inhelder, B., \& Szeminska, A. (1960). The child's conception of geometry. New York: Basic Books.

PotTs, G. R. (1974). Storing and retrieving information about ordered relationships. Journal of Experimental Psychology, 103, 431-439.

Rosch, E., \& Llovd, B. B. (Eds.) (1978). Cognition and categorization. Hillsdale, NJ: Erlbaum.

Siegel, A. W., \& White, S. H. (1975). The development of spatial representations of large-scale environments. In H. W. Resse (Ed.), Advances in child development and behavior (Vol. 10, pp. 9-55). New York: Academic Press.

STEA, D. (1976). Environmental mapping. Milton Keynes, England: The Open University Press.

Stevens, A., \& Coupe, P. (1978). Distortions in judged spatial relations. Cognitive Psychology, 10, 422-437.

Trabasso, T. (1977). The role of memory as a system in making transitive inferences. In R. V. Kail \& J. W. Hagen (Eds.), Perspectives on the development of memory and cognition (pp. 333-366). Hillsdale, NJ: Erlbaum.

WILTON, R. N. (1979). Knowledge of spatial relations: The specification of the information used in making inferences. Quarterly Journal of Experimental Psychology, 31. 133-146.

(Manuscript received April 16, 1984; revision accepted for publication January 23, 1985. . 